\title{
Research
}

\section{Primary care consultation length by deprivation and multimorbidity in England:}

\author{
an observational study using electronic patient records
}

\begin{abstract}
Background

Longer GP consultations are recommended as one way of improving care for people with multimorbidity. In Scotland, patients who are multimorbid and living in deprived areas do not have longer consultations, although their counterparts in the least deprived areas do. This example of the inverse care law has not been examined in England.
\end{abstract}

Aim

To assess GP consultation length by

socioeconomic deprivation and multimorbidity.

\section{Design and setting}

Random sample of 1.2 million consultations from 1 April 2014 to 31 March 2016 for 190036 adults in England drawn from the Clinical Practice Research Datalink.

\section{Method}

Consultation duration was derived from time of opening and closing the patient's electronic record. Mean duration was estimated by multimorbidity level and type, adjusted for number of consultations and other patient and staff characteristics and patient and practice random effects

\section{Results}

Consultations lasted 10.9 minutes on average and mean duration increased with number of conditions. Patients with $\geq 6$ conditions had $0.9(95 \%$ confidence interval $[\mathrm{Cl}]=0.8$ to 1.0$)$ minutes longer than those with none. Patients who are multimorbid and with a mental health condition had $0.5(\mathrm{Cl}=0.4$ to 0.5$)$ minutes longer than patients who were not multimorbid. However, consultations were $0.5(\mathrm{Cl}=0.4$ to 0.5 minutes shorter in the most compared with the least deprived fifth of areas at all levels of multimorbidity.

\section{Conclusion}

GPs in England spend longer with patients who have more conditions, but, at all multimorbidity levels, those in deprived areas have less time per GP consultation. Further research is needed to assess the impact of consultation length on patient and system outcomes for those with multimorbidity.

\section{Keywords}

England; mental health; multimorbidity: primary health care; referral and consultation; socioeconomic factors

\section{INTRODUCTION}

Multimorbidity is defined as the coexistence of $\geq 2$ conditions within an individual. Prevalence estimates depend on the conditions counted but recent studies suggest that around $23-27 \%$ in the general population ${ }^{1,2}$ - an estimated 14.2 million people in England ${ }^{3}$ - are affected, and that prevalence is increasing across the UK. ${ }^{4}$ The risk of multimorbidity increases with advancing age and is strongly linked to socioeconomic position, occurring more frequently and $10-15$ years earlier in the most deprived compared with the least deprived areas. ${ }^{2}$ Living with multimorbidity can be challenging, and may result in poor quality of life and difficulties with everyday activities. ${ }^{5,6}$ People with multimorbidity often require significant time and interaction with health services. Providing care to these individuals can be challenging because of the complexity of intersecting health and care requirements. ${ }^{7}$ In addition, around $30 \%$ of people who are multimorbid have both physical and mental health conditions, rising to $>40 \%$ in the most deprived onefifth of areas. ${ }^{2}$ People with comorbid physical and mental conditions have more complex care needs, and can find it more difficult to manage their conditions. ${ }^{8}$

Compared with people who are not multimorbid, people with multimorbidity require more input from the healthcare system. They require a higher number of GP consultations and have an increased likelihood of an emergency admission to hospital. 1.9 There is, however, some

A Gopfert, MBBS, BSc(Hons), public health registrar, Oxford University Hospitals, Oxford. SR Deeny, PhD, assistant director, Data Analytics; R Fisher, MA (Cantab), MB BChir, MRCP,

MRCGP, PGDip Health Res, senior policy fellow;

M Stafford, PhD, principal data analyst, the Health Foundation, London.

Address for correspondence

Mai Stafford, the Health Foundation, 8 Salisbury evidence that, if a person is more able to manage their multiple health conditions independently, they have fewer emergency admissions. 9.10 One study in an area of high deprivation showed that more time for complex consultations is associated with increased patient enablement, that is, ability to self-manage conditions. ${ }^{11}$ The Royal College of General Practitioners (RCGP), based on this premise, recommend longer consultations for patients with multimorbidity in order to reduce workload on the broader NHS. ${ }^{12}$ Likewise, people living with multimorbidity have identified longer primary care appointments as an optimal way of improving the quality of their care. ${ }^{13}$

Despite these recommendations, research in Scotland has shown that the greater need of patients with multimorbidity living in the most deprived quarter of areas is not reflected in longer consultation length. This contrasts with the least deprived quarter of areas where those with multimorbidity received longer consultations than those without..$^{14}$ This is an example of the inverse care law, where the availability of good medical care tends to vary inversely with need, and can result in unmet need for health care. Research in Scotland has also demonstrated that, although consultation rates increase with deprivation, the social gradients in multimorbidity are much steeper, indicating potentially unmet need. The authors of the present study are not aware of any research examining whether this particular example

Square, London EC4Y 8AP, UK.

Email: mai.staffordahealth.org.uk

Submitted: 27 January 2020; Editor's response: 23 April 2020; final acceptance: 6 August 2020. (C)The Authors

This is the full-length article (published online 15 Dec 2020) of an abridged version published in print. Cite this version as: Br J Gen Pract 2020; DOI: https://doi.org/10.3399/bjgp20X714029 


\section{How this fits in}

The vision of the Royal College of General Practitioners is that GPs will have more time to care for patients. Longer consultations are recommended where people need this, including people with multimorbidity. The risk of having multimorbidity is higher in more socioeconomically deprived areas. But, despite need being greatest in the most deprived areas of the UK, the number of GPs is falling fastest in these areas. Several studies have shown that GP consultations are shorter in more deprived areas. A study set in Scotland has shown that patients with multimorbidity had longer consultations with their GP, but only if they were living in the least deprived quarter of areas and not if they were living in the most deprived quarter of areas. The present study, set in England, confirms that consultations are shorter in more deprived areas. It also shows, however, that people with multimorbidity have longer consultations than those who do not have multimorbidity, and that this applies in both deprived and less deprived areas.

of the inverse care law also applies in England, although consultation length has been found to be shorter in more deprived areas. ${ }^{15,16}$

This research studied the association between GP consultation length and presence ofmultimorbidityorsocioeconomic deprivation in England. It tested whether the difference in consultation length for patients with and without multimorbidity varied between more and less deprived areas in England. Whether these factors were affected by multimorbidity type was also assessed.

\section{METHOD}

Data were obtained from the Clinical Practice Research Datalink (CPRD), a research database of anonymised patient records covering approximately $6.9 \%$ of the UK population. ${ }^{17}$ This dataset consisted of a random sample of people in England ( $n=300000$ ) eligible for linkage to an area-based measure of socioeconomic deprivation, and who were registered between 1 April 2014 and 31 March 2016 (or died during this period) in an up-tostandard practice (that is, a quality indicator based on continuous recording of patient data and completeness of recorded deaths). Consultations over this 2-year follow-up period were included. For this study, those aged $<18$ years were excluded.

\section{Consultation duration}

Consultation duration was captured in whole minutes and derived from the opening and closing time for a patient's electronic patient record. Only face-to-face consultations with a GP or GP registrar were analysed. Consultations were excluded where the record was opened for administrative purposes, telephone consultations lowing to the large number which may be triage appointments followed by face-to-face consultations), or home visit consultations las the recorded duration would only represent the time taken to record the consultation after it has ended). Consultations recorded as lasting $>60$ minutes were truncated at 60 minutes, as these were considered unlikely to reflect actual consultation length. ${ }^{18}$ Consultations recorded as lasting 0 minutes were set to 0.5 minutes. ${ }^{15}$

For the main analysis, consultations of duration $<2$ minutes were excluded, as it was deemed that these may not reflect accurate consultation length. In sensitivity analysis, all consultations were included irrespective of duration.

\section{Multimorbidity status}

The presence or absence of 36 conditions was derived at the beginning of followup on 1 April 2014. These 36 conditions were identified in previous work because they are likely to be chronic, related to reduced quality of life and mortality risk, have substantial need for ongoing treatment, ${ }^{1}$ and use publicly available lists ${ }^{19}$ for Read codes (that is, codes used by UK primary care practitioners to record information about diagnoses) and product codes (that is, codes specific to CPRD to record information about pharmacological and non-pharmacological products). Patients were grouped according to the number of conditions they had. In addition, patients were grouped into: those with 0 or 1 condition; those with $\geq 2$ conditions including $\geq 1$ mental health condition Idepression or anxiety, anorexia or bulimia, alcohol problems, other psychoactive substance use, schizophrenia), which are referred to as multimorbid - including a mental health condition'; and those with $\geq 2$ physical health conditions, which are referred to as 'multimorbid - physical only'.

\section{Socioeconomic deprivation}

Deprivation was based on the patient's area of residence (Lower Layer Super Output Area level) using deciles of the 2015 Index of Multiple Deprivation (IMD)20 grouped into high deprivation (deciles 1-3), medium 


\section{Table 1. Characteristics of included patients $(N=190036)$}

$\%(n)$

\begin{tabular}{lc}
\hline Sex & \\
Males & $45.3(86106)$ \\
Females & $54.7(103930)$ \\
\hline Age lyears) & \\
$18-29$ & $14.5(27551)$ \\
$30-39$ & $14.7(27913)$ \\
$40-49$ & $18.8(35699)$ \\
$50-59$ & $18.1(34483)$ \\
$60-69$ & $15.9(30399)$ \\
$70-79$ & $11.3(21383)$ \\
$\geq 80$ & $6.6(12608)$ \\
\hline
\end{tabular}

IMD

Quintile 1 (least deprived)

$25.9(49$ 183)

Q2

$21.1(40057)$

20.3 (38 582)

Q4

$18.4(34900)$

Q5 (most deprived)

$14.4(27314)$

\section{Multimorbidity level}

0 conditions

$39.2(74548)$

1 condition

$25.3(48043)$

2 conditions

$14.9(28306)$

3 conditions

$9.0(17054)$

$4-5$ conditions

$8.4(15969)$

$\geq 6$ conditions

$3.2(6116)$

\section{Multimorbidity type}

Not multimorbid

$64.5(122591)$

Multimorbid - physical only

$23.1(43822)$

Multimorbid - including a mental health condition

$12.4(23623)$

${ }^{a} 0-1$ long-term condition. ${ }^{b} \geq 2$ physical conditions and no mental health conditions. ${ }^{c} \geq 2$ conditions with $\geq 1$ mental health condition. $I M D=$ Index of Multiple Deprivation. $Q=$ quintile.

\section{Statistical analysis}

Multilevel linear regression analysis was conducted with consultation length as the dependent variable. Patient sex and age, number of GP consultations in the 2-year follow-up period, GP trainee status, GP sex, urban-rural classification, IMD, and multimorbidity level were controlled for. Three-level regression models accounted for the non-independence of multiple consultations within patients, and patients within practices. Additionally, an interaction between IMD and multimorbidity was tested for.

Consultation length is not normally distributed, but previous studies ${ }^{15}$ have analysed it using means and multilevel linear regression models. In sensitivity analysis, the regression models were repeated using multilevel Poisson regression. The direction and statistical significance of the associations of interest were unchanged. Therefore, the linear regression results are presented here.

\section{RESULTS}

The original sample of patients aged $\geq 18$ contained data on 2553413 face-to-face consultations, of which 1522128 were with a GP or GP registrar. Of these, 263209 lasted <2 minutes. The main analysis was conducted based on 1258919 consultations for 190036 patients lasting $\geq 2$ minutes. Consultations of duration $<2$ minutes were more common in those with more conditions $120.2 \%$ of those with $\geq 6$ conditions and $13.3 \%$ of those with no conditions) (see Supplementary Table 1 for details).

Almost $55 \%$ of the sample were females, $25.9 \%$ lived in the least deprived fifth of areas in England, and $35.5 \%$ had $\geq 2$ conditions (Table 1). A total of $23.1 \%$ had $\geq 2$ physical conditions and $12.4 \%$ had multimorbidity that included $\geq 1$ mental health condition.

In unadjusted analysis (Table 2), females had longer consultations (mean duration 11.0 minutes) and more consultations (mean 8.6 over 2 years) than males Imean 10.9 minutes and 6.7 consultations respectively). Older people did not have longer consultations, but they had more consultations compared with younger people. Compared with fully qualified GPs, GP registrars had longer consultations with a mean duration of 14.4 minutes. Mean consultation length was shorter for people living in the most compared with the least deprived fifth of areas (10.7 versus 11.2 minutes). Shorter consultations were also seen for patients who were not multimorbid (10.8 minutes compared with 
Table 2. Consultations by sociodemographic characteristics ( $N=1258$ 919)

\begin{tabular}{|c|c|c|c|c|c|c|}
\hline & & \multicolumn{3}{|c|}{$\begin{array}{l}\text { Duration of consultations } \\
\text { (includes consultations } \\
\quad \geq 2 \text { minutes) }\end{array}$} & \multicolumn{2}{|c|}{$\begin{array}{c}\text { Number of consultations } \\
\geq 2 \text { minutes per } \\
\text { patient over } 2 \text { years }\end{array}$} \\
\hline & & Consultations (N) & Mean & SD & Mean & SD \\
\hline \multirow[t]{2}{*}{ Sex } & Males & 492383 & 10.9 & 7.8 & 6.7 & 7.9 \\
\hline & Females & 766536 & 11.0 & 7.8 & 8.6 & 9.1 \\
\hline \multirow[t]{7}{*}{ Age lyears) } & $18-29$ & 139667 & 10.6 & 7.4 & 5.7 & 6.2 \\
\hline & $30-39$ & 154375 & 10.9 & 7.6 & 6.3 & 7.0 \\
\hline & $40-49$ & 204351 & 11.2 & 7.7 & 6.5 & 7.1 \\
\hline & $50-59$ & 216932 & 11.1 & 7.6 & 7.3 & 7.8 \\
\hline & $60-69$ & 220044 & 10.9 & 7.7 & 8.6 & 8.9 \\
\hline & $70-79$ & 192617 & 10.9 & 7.9 & 11.0 & 10.6 \\
\hline & $\geq 80$ & 130933 & 10.7 & 8.9 & 13.0 & 13.7 \\
\hline \multirow[t]{2}{*}{ GP registrar } & No (Qualified GP) & 1150219 & 10.6 & 7.5 & - & - \\
\hline & Yes & 108700 & 14.4 & 9.4 & - & - \\
\hline \multirow[t]{3}{*}{ GP sex } & Male & 666793 & 10.3 & 7.7 & - & - \\
\hline & Female & 582178 & 11.5 & 7.8 & - & - \\
\hline & Unknown & 9948 & 12.1 & 8.9 & - & - \\
\hline \multirow[t]{3}{*}{ Rural-urban classification } & Rural & 160824 & 10.7 & 7.9 & 8.0 & 9.1 \\
\hline & Urban city & 656125 & 11.0 & 7.6 & 7.6 & 8.4 \\
\hline & Urban conurbation & 441970 & 11.0 & 8.0 & 7.8 & 8.8 \\
\hline \multirow[t]{5}{*}{ IMD } & Q1 (least deprived) & 318041 & 11.2 & 7.9 & 7.6 & 8.5 \\
\hline & Q2 & 258653 & 11.0 & 7.8 & 7.5 & 8.8 \\
\hline & Q3 & 258678 & 10.9 & 7.8 & 7.8 & 8.7 \\
\hline & Q4 & 236385 & 10.8 & 7.8 & 7.9 & 8.5 \\
\hline & Q5 (most deprived) & 187162 & 10.7 & 7.6 & 8.0 & 78.5 \\
\hline \multirow[t]{6}{*}{ Multimorbidity level } & 0 conditions & 312485 & 10.8 & 7.3 & 4.3 & 4.6 \\
\hline & 1 condition & 286130 & 10.9 & 7.5 & 7.0 & 6.5 \\
\hline & 2 conditions & 218395 & 10.9 & 7.7 & 9.2 & 8.1 \\
\hline & 3 conditions & 162367 & 11.0 & 8.0 & 11.5 & 10.2 \\
\hline & 4-5 conditions & 187229 & 11.0 & 8.2 & 14.4 & 13.1 \\
\hline & $\geq 6$ conditions & 92313 & 11.2 & 8.9 & 18.7 & 18.1 \\
\hline \multirow[t]{4}{*}{ Multimorbidity type } & Not multimorbida & 598615 & 10.8 & 7.4 & 5.6 & 4.8 \\
\hline & $\begin{array}{l}\text { Multimorbid } \\
\text { Of which: }\end{array}$ & 660304 & 11.0 & 8.1 & 11.8 & 11.5 \\
\hline & Multimorbid - physical only ${ }^{b}$ & 397098 & 10.9 & 8.0 & 11.0 & 10.3 \\
\hline & $\begin{array}{l}\text { Multimorbid - including } \\
\text { a mental health condition }\end{array}$ & 263206 & 11.1 & 8.3 & 13.4 & 13.2 \\
\hline
\end{tabular}

${ }^{a} 0-1$ long-term condition. ${ }^{b} \geq 2$ physical conditions and no mental health conditions. ${ }^{c} \geq 2$ conditions with $\geq 1$ mental health condition. IMD = Index of Multiple Deprivation. $Q=$ quintile. SD = standard deviation.

11.0 for patients who are multimorbid). Among patients who are multimorbid, those with $\geq 1$ mental health condition had mean consultation time of 11.1 minutes and those with only physical health conditions 10.9 minutes.

Table 3 summarises estimates from the regression models. In the main analysis limited to consultations lasting $\geq 2$ minutes, controlling for patient and staff characteristics, residence in a more deprived area was associated with a shorter consultation. Mean duration was $0.46195 \%$ confidence intervals $[\mathrm{Cl}]=0.40$ to 0.53 ) minutes shorter for those in the most compared with the least deprived fifth of areas. Consultation length increased with number of conditions the patient had and was $0.94(\mathrm{Cl}=0.84$ to 1.03$)$ minutes longer for those with $\geq 6$ compared with no long-term conditions. Consultation length also depended on multimorbidity type, with patients with $\geq 2$ physical conditions having 0.30 minutes longer with the GP and those with $\geq 2$ conditions including a mental health condition having 0.47 minutes longer compared with patients who were not multimorbid (Table 4).

No clear evidence was found that the association between multimorbidity level or type and consultation length was different for patients in more versus less deprived 


\section{Table 3. Association ${ }^{a}$ between consultation duration and multimorbidity level and area deprivation}

\begin{tabular}{|c|c|c|c|c|}
\hline & \multicolumn{2}{|c|}{ Consultations $\geq 2$ mins, $N=1258919$} & \multicolumn{2}{|c|}{ All consultations, $N=1522128$} \\
\hline & $\begin{array}{c}\text { Regression } \\
\text { coefficient }\end{array}$ & $\mathrm{Cl}$ & $\begin{array}{c}\text { Regression } \\
\text { coefficient }\end{array}$ & $\mathrm{Cl}$ \\
\hline \multicolumn{5}{|l|}{ IMD } \\
\hline Q1 (least deprived) & Ref & & Ref & \\
\hline Q2 & -0.18 & $(-0.23 \text { to }-0.13)^{b}$ & -0.12 & $(-0.17 \text { to }-0.06)^{t}$ \\
\hline Q3 & -0.20 & $(-0.26 \text { to }-0.15)^{b}$ & -0.12 & $(-0.18 \text { to }-0.07)^{4}$ \\
\hline Q4 & -0.31 & $(-0.36 \text { to }-0.24)^{b}$ & -0.23 & $(-0.29$ to -0.17$)$ \\
\hline Q5 (most deprived) & -0.46 & $(-0.53 \text { to }-0.40)^{b}$ & -0.27 & $(-0.34$ to -0.21$)$ \\
\hline \multicolumn{5}{|l|}{ Multimorbidity level } \\
\hline 0 & Ref & & Ref & \\
\hline 1 & 0.07 & $(0.02 \text { to } 0.12)^{c}$ & -0.08 & $(-0.13$ to -0.03$)$ \\
\hline 2 & 0.22 & $(0.17 \text { to } 0.28)^{b}$ & 0.01 & (-0.05 to 0.07$)$ \\
\hline 3 & 0.45 & $(0.38 \text { to } 0.52)^{\mathrm{b}}$ & 0.21 & $(0.14 \text { to } 0.28)^{b}$ \\
\hline $4-5$ & 0.67 & $(0.60 \text { to } 0.74)^{\mathrm{b}}$ & 0.45 & $(0.37 \text { to } 0.52)^{b}$ \\
\hline$\geq 6$ conditions & 0.94 & $(0.84 \text { to } 1.03)^{\mathrm{b}}$ & 0.77 & $(0.66 \text { to } 0.87)^{b}$ \\
\hline
\end{tabular}

Three-level regression model (consultations nested within patients within practices) includes patient sex, age, number of consultations per year, GP trainee status, GP sex, urban-rural classification, multimorbidity level, and $I M D .{ }^{b P}<0.001 . \cdot P<0.05 . C l=$ confidence interval. $I M D=$ Index of Multiple Deprivation. $Q=$ quintile. Ref = the category against which the other categories are compared in the statistical model.

\section{Table 4. Association ${ }^{\text {a }}$ between consultation duration and multimorbidity type and area deprivation}

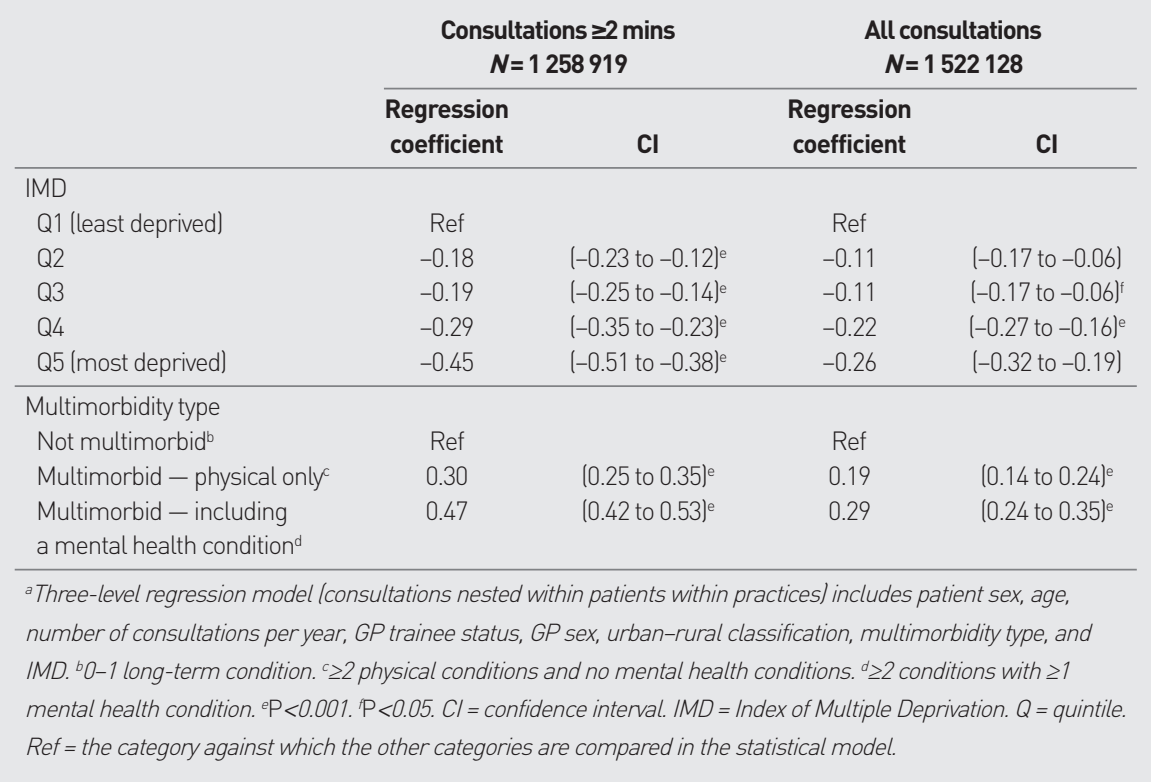

areas. Figure 1 shows consultation duration by IMD and multimorbidity type from the model, including the interaction of these two factors. It illustrates that, for all multimorbidity types, patients in the most deprived areas had shorter consultations than those in the least deprived areas. It also illustrates that the mean consultation length for a patient without multimorbidity in a low deprivation area (10.9 minutes) was the same as that for a multimorbid patient with physical and mental health conditions in an area of high deprivation.

The same patterns were found when all consultations lincluding those lasting $>2$ minutes) were analysed. Regression estimates show smaller differences in consultation length by multimorbidity level when all consultations were included. This was expected because very short consultations were more common in patients with more long-term conditions.

\section{DISCUSSION \\ Summary}

Living in an area of high socioeconomic deprivation is associated with shorter GP consultations. GP consultation length increased with increasing number of health conditions. Consultations were also longer for multimorbid patients with a mental health condition than for multimorbid patients with physical conditions only. The positive association between consultation length and number of health conditions was seen in both deprived and less deprived areas.

\section{Strengths and limitations}

A strength of this study was the large sample size and the use of routine data to minimise selection bias. The use of multilevel regression analysis allowed for unobserved similarities between practices and between patients that could affect consultation duration. The association between deprivation and duration remained on adjustment for total number of consultations, indicating that use of additional consultations did not explain the shorter consultations in more deprived areas. This study was limited by several factors. CPRD data provide consultation time based on the open and close time of the electronic record. This is the amount of time a practitioner had the file open, which may be affected by other factors including practitioner preference regarding whether to complete and close a record later in the day or while the patient is present, and the possibility of clinicians forgetting to close a consultation until after the care episode has ended lalthough all consultations were capped at a maximum of 60 minutes). There is, however, no evidence to suggest that these factors differ by patient level of deprivation or multimorbidity. Previous analysis used video-recording to accurately capture consultation duration, although this approach may have altered GP behaviour. This study also focused on primary care 


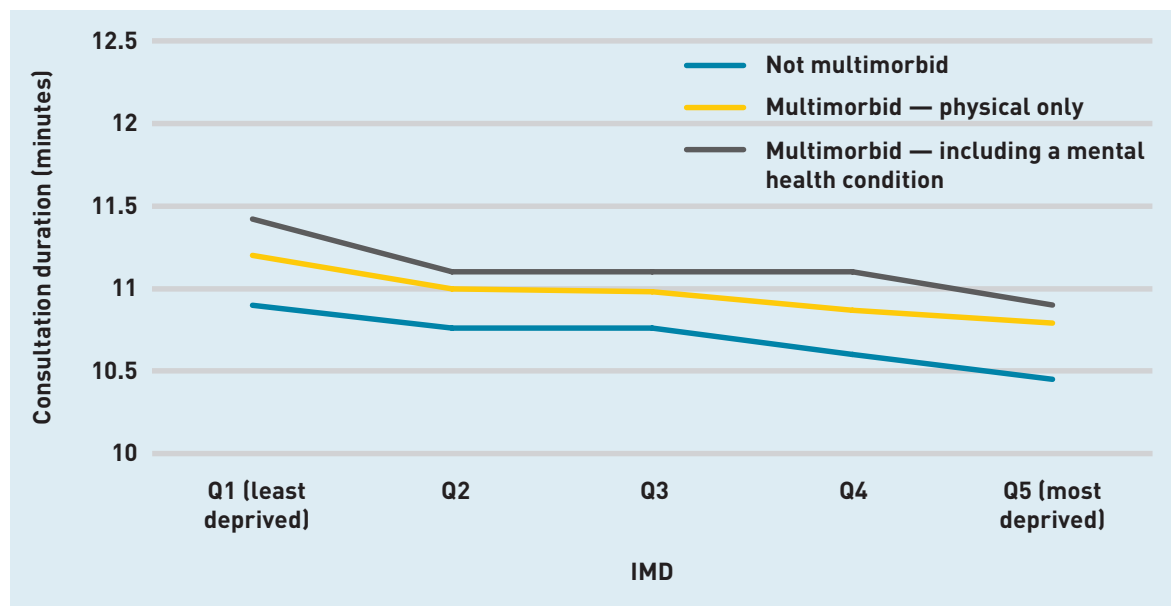

Figure 1. Consultation duration by Index of Multiple Deprivation and multimorbidity type: consultations lasting $\geq 2$ minutes. ${ }^{a}$

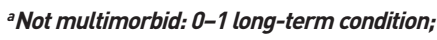
multimorbid - physical only: $\geq 2$ physical conditions and no mental health conditions; multimorbid - including a mental health condition: $\geq 2$ conditions with $\geq 1$ mental health condition. IMD = Index of Multiple Deprivation. $Q=$ quintile. delivered by GPs. Future analysis should also consider consultations with nurses, because nurses provide a sizeable proportion of primary care for people with multimorbidity. ${ }^{18}$ As primary care only forms a single component of health care for people with multimorbidity, further studies should also consider care provided within hospitals and other parts of the health system.

The adjusted difference in consultation time for patients in the most compared with the least deprived areas amounted to 0.5 minutes. The magnitude of this difference appears small, and further work is needed to quantify associations between consultation length and patient experience, outcomes, or use of other health services, as others have noted. ${ }^{21}$ This small value should, however, be interpreted in the context of an average consultation of just under 11 minutes.

\section{Comparison with existing literature}

This paper adds to the evidence that multimorbidity and deprivation influence consultation time with a GP. Particularly concerning is ongoing evidence indicating that patients in deprived areas have shorter consultation times. ${ }^{15}$ These findings support evidence previously found in Scotland based on video-recorded consultations to provide an accurate measure of time spent with patients. ${ }^{14}$ That study considered a single consultation for each patient, whereas the present study adds to their findings in showing that a similar pattern is observed (that is, shorter consultations for patients in more deprived areas) across multiple consultations over a 2-year period of usual care. This is likely to reflect ongoing job pressures for GPs in deprived areas, and a greater need for care among this group of patients. Practices in deprived areas tend to have lower levels of GP staffing. ${ }^{22}$ The staffing level and patient load at a particular GP surgery influences the work pressure for GPs, and can therefore influence the consultation time available. ${ }^{22}$ Given these pressures, other factors that could affect patient experience and patient outcomes, such as continuity of care or GP empathy, may also differ by deprivation level, as has been found in Scotland. ${ }^{15}$ These other characteristics of the consultation were not examined, and further work is needed to explore those factors and to test the contribution to outcomes of consultation length, continuity of care, and patient experiences of the consultation. The previous study set in Scotland compared consultation length for practices in high- and low-deprivation areas, whereas the present study used deprivation in the patient's local area. Although patient and practice deprivation will be positively correlated, they may influence consultation length independently via different mechanisms. Further analysis including deprivation at both patient and practice level would be useful, but was not possible with the current data.

It was also identified that patients with multimorbidity receive longer consultations. Consultation length increased with the number of conditions a patient had. This is in line with calls for longer GP consultations for patients who are multimorbid; however, whether these relatively small differences in consultation length are related to, or sufficient to achieve, better patient outcomes remains to be tested. Previous evidence from Scotland ${ }^{14}$ showed that patients with multimorbidity received around 3 minutes longer with their GP than those without multimorbidity in affluent areas, but that this was not the case in deprived areas. In England, patients with multimorbidity in both more and less deprived areas had longer consultations than their nonmultimorbid counterparts.

GPs in England are spending longer with patients who have more long-term conditions. The consultation length also depends on the types of conditions the patient has. Having a mental health condition can make it more difficult to manage complex care needs, and longer consultations have been linked to better handling of psychological problems in primary care. ${ }^{23,24}$ This analysis shows that multimorbidity including a mental health condition was associated with having a longer consultation compared with having multiple physical conditions and compared with not having multimorbidity. However, 
the analysis also shows that this additional time is counteracted by living in a deprived area. The association between deprivation and consultation length is equal in magnitude and opposite in effect to the association between multimorbidity and consultation length. This means that a patient with multiple mental and physical health conditions living in an area of high deprivation receives the same amount of time with their GP as a person without multimorbidity in an area of low deprivation.

\section{Implications for research and practice}

This study shows that the inverse care law is alive and well in general practice in England. Not only do people living in more deprived areas of the country have on average shorter GP appointments, the same pattern is observed even when those people have multiple health conditions. This suggests that there could be unmet need among patients with complex care needs, particularly patients living in deprived areas with both mental and physical health conditions.

Understanding why shorter consultation times in general practice are observed in areas of high deprivation is crucial to understanding how this could be changed. This includes understanding patient factors as well as those related to the organisation and delivery of general practice.

Undersupply of GPs relative to population need and corresponding higher workload may be a key driver of shorter consultation times, and evaluation of the impact of initiatives encouraging GPs to train and work in underdoctored areas is awaited. Increasing skill mix in primary care by recruiting additional allied health professionals is seen as one way of freeing up GP time to focus on more complex patients. These staff may also directly contribute to and improve care for people with multimorbidity. Initiatives to ensure these additional staff will be distributed equitably across the country and to encourage them to work in areas of high deprivation will be needed. If additional staff gravitate to areas of lower deprivation, then there will be paradoxically even fewer staff relative to need in the areas of highest deprivation. ${ }^{22}$

The positive association between consultation length and number of longterm conditions that were identified is in line with calls for longer GP consultations for patients who are multimorbid, although whether these relatively small differences in consultation length are related to, or sufficient to achieve, better patient outcomes remains to be tested.

\section{Funding}

This study was funded by the Health Foundation as part of core activity of members of staff at the Health Foundation.

\section{Ethical approval}

Routinely collected, retrospective, anonymised data were used for this analysis. Approval to use the data was granted by the Independent Scientific Advisory Committee (CPRD protocol number ISAC17_150RMn2).

\section{Provenance}

Freely submitted; externally peer reviewed.

\section{Competing interests}

The authors have declared no competing interests.

\section{Acknowledgements}

We thank the Data Management Team at the Health Foundation for their work in preparing the data.

\section{Open access}

This article is Open Access: CC BY 4.0 licence (http://creativecommons.org/ licences/by/4.0/).

\section{Discuss this article}

Contribute and read comments about this article: bjgp.org/letters 


\section{REFERENCES}

1. Cassell A, Edwards D, Harshfield A, et al. The epidemiology of multimorbidity in primary care: a retrospective cohort study. Br J Gen Pract 2018; DOI: https://doi. org/10.3399/bjgp18X695465

2. Barnett K, Mercer SW, Norbury M, et al. The epidemiology of multimorbidity in a large cross-sectional dataset: implications for health care, research and medical education. Lancet 2012; 380(9836): 37-43.

3. Stafford M, Steventon A, Thorlby R, et al. Understanding the health care needs of people with multiple health conditions. London: Health Foundation, 2018. https://www.health.org.uk/publications/understanding-the-health-care-needsof-people-with-multiple-health-conditions (accessed 16 Nov 2020).

4. Kingston A, Robinson L, Booth $\mathrm{H}$, et al. Projections of multi-morbidity in the older population in England to 2035: estimates from the Population Ageing and Care Simulation (PACSim) model. Age Ageing 2018; 47(3): 374-380.

5. Kanesarajah J, Waller M, Whitty JA, Mishra GD. Multimorbidity and quality of life at mid-life: a systematic review of general population studies. Maturitas 2018; 109: 53-62.

6. Williams JS, Egede LE. The association between multimorbidity and quality of life, health status and functional disability. Am J Med Sci 2016; 352(1): 45-52.

7. Mercer SW, Watt GC. The inverse care law: clinical primary care encounters in deprived and affluent areas of Scotland. Ann Fam Med 2007; 5(6): 503-510.

8. Moffat K, Mercer SW. Challenges of managing people with multimorbidity in today's healthcare systems. BMC Fam Pract 2015; 16(1): 129

9. Frølich A, Ghith N, Schiøtz M, et al. Multimorbidity, healthcare utilization and socioeconomic status: a register-based study in Denmark. PloS One 2019; 14(8): e021418.

10. Deeny S, Thorlby R, Steventon A. Reducing emergency admissions: unlocking the potential of people to better manage their long-term conditions. London: Health Foundation, 2018. https://www.health.org.uk/publications/reducingemergency-admissions-unlocking-the-potential-of-people-to-better-managetheir-long-term-conditions laccessed 16 Nov 2020)

11. Mercer SW, Fitzpatrick B, Gourlay G, et al. More time for complex consultations in a high-deprivation practice is associated with increased patient enablement. Br J Gen Pract 2007; 57(545): 960-966.

12. Royal College of General Practitioners. Responding to the needs of patients with multimorbidity. A vision for general practice. London: RCGP, 2016. https://
wuw.rcgp.org.uk/-/media/Files/Policy/A-Z-policy/RCGP-Responding-to-needsof-Multimorbitiy-2016.ashx?la=en (accessed 16 Nov 2020).

3. Mercer SW, O'Brien R, Fitzpatrick B, et al. The development and optimisation of a primary care-based whole system complex intervention (CARE Plus) for patients with multimorbidity living in areas of high socioeconomic deprivation. Chronic Illn 2016; 12(3): 165-181.

14. Mercer SW, Zhou Y, Humphris GM, et al. Multimorbidity and socioeconomic deprivation in primary care consultations. Ann Fam Med 2018; 16(2): 127-131.

15. Stevens S, Bankhead C, Mukhtar T, et al. Patient-level and practice-level factors associated with consultation duration: a cross-sectional analysis of over one million consultations in English primary care. BMJ Open 2017; 7(11): e018261.

16. McLean G, Guthrie B, Mercer SW, Watt GCM. General practice funding underpins the persistence of the inverse care law: cross-sectional study in Scotland. Br J Gen Pract 2015; DOI: https://doi.org/10.3399/bjgp15X687829.

17. Herrett E, Gallagher AM, Bhaskaran K, et al. Data resource profile: Clinical Practice Research Datalink (CPRD). Int J Epidemiol 2015; 44(3): 827-836.

18. Salisbury C, Man M-S, Bower $P$, et al. Management of multimorbidity using a patient-centred care model: a pragmatic cluster-randomised trial of the 3D approach. Lancet 2018; 392(10141): 41-50.

19. Primary Care Unit, University of Cambridge. Code lists. http://www.phpc.cam. ac.uk/pcu/cprd_cam/codelists/ laccessed 16 Nov 2020).

20. Deveugele $\mathrm{M}$, Derese $\mathrm{A}$, van den Brink-Muinen A, et al. Consultation length in general practice: cross sectional study in six European countries. BMJ 2002; 325(7362): 472

21. Wilson AD, Childs S, Goncalves-Bradley DC, Irving GJ. Interventions to increase or decrease the length of primary care physicians' consultation. Cochrane Database Syst Rev 2016; 2016(8): CD003540.

22. Gershlick B, Fisher R. A worrying cycle of pressure for GPs in deprived areas. London: Health Foundation, 2019. https://www.health.org.uk/news-andcomment/blogs/a-worrying-cycle-of-pressure-for-gps-in-deprived-areas laccessed 16 Nov 2020).

23. Freeman GK, Horder JP, Howie JGR, et al. Evolving general practice consultation in Britain: issues of length and context. BMJ 2002; 324(7342): 880-882

24. Hutton C, Gunn J. Do longer consultations improve the management of psychological problems in general practice? A systematic literature review. BMC Health Serv Res 2007: 7: 71. 